\title{
L'absurdité Dans Le Drame Caligula Albert Camus: Une Étude Selon La Theorie De L'hermeneutique De Paul Ricœur
}

\author{
Niken Nining Aninsi ${ }^{\bowtie}$ Suluh Edhi Wibowo ${ }^{\bowtie}$ Sunahrowi
}

Département de la Langue et la Littérature Étrangère, Faculté des Langues et des Arts, Universitas Negeri Semarang, Indonesia

\section{Info d'article}

Histoire de l'Atrticle : Reçu Janvier 2020 Accepté Mars 2020 Publié Mai 2020

\section{Keywords :} Absurd, Caligula, Hermeneutics

\begin{abstract}
Language is a symbolic system that contains elements called words. That is to say, a word is also a symbol, because both are present in another form. The words are arranged so that they form a text. In literary works, the text functions as an element of creation. They consist of poetry, prose, and drama. The drama is a performance that contains dialogues between the actors. In this research, the drama Caligula was chosen as a material object. Caligula is one of Albert Camus' best known masterpieces. Written in 1938, this work by Camus is well known in the context of the trilogy 'Cycle of the absurd' with the novels Le Mythe de Sisyphe et L'Etranger. This drama is presented in absurd form with a lot of significant baggage. It is for this reason that a good tool is needed to discover and analyze all these symbols. This tool is called the hermeneutical approach. In this case, it is the hermeneutic theory of Paul Ricœur which is used as a formal object to reveal the absurd senses in Camus' drama. The technique of data collection used is that of bibliography supplemented by the method of literature. While the data analysis technique used is that of content analysis, applying the descriptive analytical method. To conclude, the researcher found that the metaphor and symbols had described an emotional story through the characters and characters of the drama Caligula.
\end{abstract}

\section{Extrait}

\begin{abstract}
La langue est un système symbolique qui contient des éléments appelé les mots. C'est-à-dire, un mot est aussi un symbole, parce que tous les deux sont présents dans une autre forme. Les mots sont arrangés de sorte qu'ils forment un texte. Dans les œuvres littéraires, le texte fonctionne comme élément de la création. Elles se composent de la poésie, de la prose, et du drame. Le drame est une performance qui contient des dialogues effectués entre les acteurs. Dans cette recherche, le drame Caligula a été choisi comme objet matériel. Caligula est l'un des chefs-d'œuvres le plus connu d'Albert Camus. Écrit en 1938, cette œuvre de Camus est bien connue dans le cadre de la trilogie 'Cycle de l'absurde' avec les romans Le Mythe de Sisyphe et L'Etranger. Ce drame est présenté dans la forme absurde avec beaucoup de bagages significatifs. C'est pour cette raison-là qu'un bon outil est nécessaire pour découvrir et analyser tous ces symboles. Cet outil s'appelle l'approche herméneutique. Dans ce cas-là, c'est la théorie herméneutique de Paul Ricœur qui est utilisée comme objet formel pour révéler les sens absurdes dans le drame de Camus. La technique de la collecte de données utilisée est celle de bibliographique complétée par la méthode de la littérature. Tandis que la technique de l'analyse des données utilisée est celle de l'analyse des contenus, en appliquant la méthode descriptive analytique. Pour conclure, la chercheuse a trouvé que la métaphore et les symboles avaient décrit une histoire émotionnelle à travers les caractères et les personna ges du drame Caligula.
\end{abstract}

(C) 2020 Universitas Negeri Semarang 


\section{INTRODUCTION}

L'œuvre littéraire est un reflet des phénomènes qui se produisent dans la société. Par conséquent, son existence est une question importante et est devenue quotidienne dans la société, à la fois comme nécessité et comme divertissement.

Il y a plusieurs genres d'œuvre littéraire, ce sont la prose, la poésie et le drame. La prose est une

forme ordinaire de discours écrit ou oral qui n'est pas soumise aux règles du rythme et de la musicalité. La poésie est une forme d'essai liée par une rime, un rythme ou un nombre de lignes et marquée par une langage complète. Tandis que le drame est une histoire de la vie humaine qui est racontée sur la scène en se basant sur le scénario, qui utilise des conversations bien pensées, et des éléments supporté (les décorations, les costumes, les maquillages, les lumières, ainsi que les musiques) et dont le public est témoin (Djuharmie, 2009: 2).

Caligula est un drame écrit par Albert Camus à partir de 1938 et publié pour la première fois en

mai 1944 aux Éditions Gallimard. On peut dire que Caligula est l'une des œuvres les plus réussies de Camus. Sa première représentation en 1945 introduit Gérard Philippe. Le 26 mars 1955, Camus lui- même se présente le drame au théâtre Noctambules.

Caligula raconte un jeune empereur qui dirigeait Rome avec une ambition et une obsession dépassant la raison humaine. À cause de son ambition, il a sacrifié tout ce qu'il possédait, y compris le peuple de Rome, même sa propre maîtresse. L'empereur Caligula était à l'origine un bon dirigeant sage. Mais à cause de la morte de Drusilla, sa sœur et son être cher, il devient une figure cruelle et sadique. Il n'a pas hésité à condamner à mort tous ceux qui s'opposaient à son ordre. Jusqu'à un jour, il y a eu des rébellions qui ne supportaient pas sa cruauté, ils planent de se rebeller et d'éliminer l'empereur. À la fin de l'histoire, Caligula est mort entre les mains des rebellions.

\section{MÉTHODE DE RECHERCHE}

En examinant le drame Caligula, la chercheuse utilise l'approche objective. Ratna (2012: 61) affirme que la recherche vise la littérature elle-même. Si dans la recherche l'attention est dirigée sur l'auteur, le modèle de recherche est plus proche de l'approche expressive et inversement, si l'attention est dirigée sur le travail, le modèle de recherche est plus proche de l'approche objective.

L'approche objective est une approche qui attire l'attention sur la littérature elle-même. Cette approche considère la littérature comme une structure autonome et libre de ses relations avec la réalité, les auteurs, et les lecteurs. Wellek \& Warren (1990) considèrent également cette approche comme une approche intrinsèque, car les études sont centrées sur les éléments intrinsèques de la littérature considérés comme ayant leur propre rondeur, cohérence et vérité.

\section{RÉSULTAT ET DISCUSSION}

Cette analyse est divisée en deux parties, ce sont l'interpretation selon la théorie herméneutique et la divulgation de l'absurdité. Dans la première partie, l'approche herméneutique, il existe deux étapes d'analyse, le métaphore et le symbole. Tandis que la seconde partie comporte trois étapes d'analyse, l'absurdité tout d'abord, ensuite c'est la mort, et la révolte à la fin.

\section{Métaphores dans le drame Caligula}

CESONIA (C/I/52)

\section{Mais Rome tout entière voit Caligula partout. Et Caligula, en effet ne voit que son idée.}

Cette phrase contient une métaphore phrastique, parce que le symbole entier de l'impression se trouve dans la phrase entière. La phrase dans cette pièce montre que tous les habitants de Rome étaient inquiets pour Caligula, mais Caligula s'en fichait, il était seulement occupé à être obsédé par ses propres pensées.

HÉLICON (C/II/98)

C'est dangereux, ma colombe. 
Cette phrase contient une métaphore complémentative, parce que tout le symbole de l'impression se trouve dans l'objet de la phrase: "ma colombe". Le mot "ma colombe" dans cette pièce illustre la belle et obéissante silhouette de Scipion aux yeux d'Hélicon. De plus, Scipion est également un écrivain littéraire. Le mot pigeon est donc très approprié pour décrire sa personnalité.

\section{Symboles dans la drame Caligula}

\section{Dix de retrouvées}

Trouvé dans la ronde I, scène 1 (39)

Bien sûr! Une de perdue, dix de retrouvées

Le mot "dix de retrouvées" dans la phrase précédente symbolise la perte de quelque chose, il sera remplacé par quelque chose de meilleur ou de plus nombreux. Le charme montre une attitude inflexible et optimiste face à l'adversité.

\section{Maison publique}

Trouvé dans la ronde II, scène 1 (70)

Octavius, il a enlevé ta femme et la fait travailler maintenant dans sa maison publique.

Le mot "maison publique" dans la phrase ci-dessus symbolise le lieu de la prostitution. La phrase montre que Caligula était très arbitraire envers son peuple. Il a intentionnellement amené les femmes au lit ou a travaillé dans la maison close qu'il possédait. Les maris ne se sont opposés pas à cette contrainte, de peur que la menace d'un châtiment ne soit acceptée.

\section{L'absurdité}

(1)

C/I/48

C: Oui. Enfin! mais je ne suis pas fou et même je n'ai jamais éte aussi raisonnable. simplement, Je me suis senti tout d'un coup un besoin d'impossible. ...

\section{$\mathrm{H}:$ C'est une opinion assez répandue.}

C : Il est vrai. Mais je ne le savais pas auparavant. Ce monde tel, qu'il est fait, n'est pas supportable. J'ai donc besoin de la lune, ou du bonheur, ou de l'immortalité, de quelque chose qui soit dement peutêtre, mais qui ne soit pas dece monde.

Avoir un pouvoir est l'une des faveurs du monde qui sera toujours accueillie à bras ouverts. De même avec Caligula. Lui, avec son pouvoir, peut facilement obtenir tout ce qu'il veut. Peutêtre juste assez pour effleurer les doigts, le souhait sera présenté devant vos yeux. Mais pas cette fois, le désir qu'il exprimait était si irrationnel et semblait ridicule. Cela rend donc la main droite confuse et encore confuse. Le vouloir difficile du roi est vu par Helicon comme une simple hallucination.

À en juger par la citation du dialogue que la chercheuse a énuméré à la page précédente, l'absurdité de Caligula est présentée dans la première phrase. Caïus - l'appel de Caligula - a prétendu qu'il n'était pas fou, même si en réalité, il n'y avait personne en bonne santé qui pensait à faire une déclaration pour obtenir la reconnaissance de sa santé mentale. Par cette phrase, on peut interpréter que Caligula n'était plus sain d'esprit à partir de ce moment. Cette spéculation est encore renforcée dans la phrase suivante où il exprime consciemment son désir d'avoir quelque chose d'impossible: la lune.

Dans la simple citation: "J'ai envoyé un tout dun coup un besoin d'impossible", il était clair que Caligula avait reconnu l'absurdité qui était en lui. Il était bien conscient que la chose à laquelle il pensait était une impossibilité. Néanmoins, il n'a jamais arrêté son intention. Il est resté confiant et l'a exprimé à tout le palais. Il ne fuyait pas l'absurdité, mais le gardait dans sa conscience et faisait délibérément face à la futilité. Il est clair que Camus implique une forme de "décision morale" reflétée 
par la figure de Caligula, c'est la rébellion en cours menée par les humains pour faire face à la réalité d'un monde plein de validité.

De plus, la phrase: "J'ai donc besoin de la lune, ou du bonheur, de l'immortalité, de quelque chose qui soit dément peut-être", Caligula explique la raison et les autres impossibilités dont il a rêvé. Indirectement, il a demandé à d'autres personnes de le soutenir, ce qui l'a rendu encore plus confiant dans ce désir fou. Le monde et toutes ses incertitudes sont capables de tromper l'esprit humain et de le transformer en une hallucination absurde et irrationnelle. La pensée de l'absurdité découle de la recherche par l'homme d'une compréhension globale de la vie qui s'avère incompréhensible.

\section{La mort}

(7)

\section{C/II/ 88-89}

C: Soyons généreux, Hélicon! Découvrons-leur nos petits secrets. Allez, section III, paragraphe premier.

$H$ :"L'exécution soulage et délivre. Elle est universelle, fortifiante et juste dans ses applications comme dans ses intentions. On meurt parce qu'on est coupable. On est coupable parce qu'on est sujet de Caligula. Or, tout le monde est sujet de Caligula. Donc, tout le monde est coupable. D'où il ressort que tout le monde meurt. C'est une question de temps et de patience".

$C$ : Qu'en pensez-vous? La patience, hein, voilà une trouvaille! Voulez-vous que je vous dise : c'est ce que j'admire le plus en vous. Maintenant, messieurs, vous pouvez disposer. Cherea n'a plus besoin de vous. Cependant, que Coesonia reste! Et Lepidus et Octavius! Mereia aussi. Je voudrais discuter avec vous de l'organisation de ma maison publique. Elle me donne de gros soucis.

La citation ci-dessus montre que la cause fondamentale du problème est la publication du règlement sur la peine de mort à appliquer. Caligula a expliqué qu'il n'y avait pas de négociation pour quiconque avait commis une erreur. Les règles établies s'appliquent à tous dans tous les milieux. Ainsi, tous les habitants de Rome subiront leur propre mort à tour de rôle, seulement ils devront "être un peu patients" dans l'attente du moment, comme expliqué dans la phrase: "On meurt parce qu'on est coupable. On est coupable parce qu'on est sujet de Caligula. Or, tout le monde est sujet de Caligula. Donc, tout le monde est coupable. D'où il ressort que tout le monde meurt. C'est une question de temps et de patience".

La mort est l'un des facteurs qui influencent la pensée de Camus sur l'absurdité. La mort est la frontière de la vie qui implique qu'en présence d'absurdité. La conscience humaine n'est qu'une incompétence et une contradiction, ce qui devient une condition quotidienne de l'être humain.

\section{La révolte}

(11)

\section{$\mathrm{C} / \mathrm{I} / 59-60$}

Ce : Je surpris, Caïus.

$C:$ Ne sois pas surpris. Je n'aime pas littérateurs et je ne peux supporter leurs mensonges. Ils parlent pour ne pas s'écouter. S'ils s'écoutaient, ils sauraient qu'ils ne sont rien et ne pourraient plus parler. Allez, rompez, j'ai horreur de faux témoins.

Ce: Si nous mentons, c'est souvent sans le savoir. Je plaide non coupable.

Après avoir été confiné pendant des jours, Caligula a apparu et Cherea l'a salué. Malheureusement, Caligula n'était pas très content de la présence prémature de Cherea. Cherea était surprise. Il était sur de ne pas avoir commis une erreur qui aurait pu rendre Caligula mécontent. Ce dernier a commencé apparemment à placer des soupçons, comme ce qui est expliqué dans la phrase : "Je n'aime pas littérateurs et je ne peux supporter leurs mensonges". Selon lui, Cherea lui avait menti et il ne pouvait plus se fier à ses paroles. En tant qu'écrivain, il existe certainement de nombreuses œuvres de Cherea qui ne sont qu'imaginaires. Il n'y a pas de faits réels et des preuves peuvent être trouvées 
dans le monde réel. Par conséquent, le cachet en tant que "faux témoin" était directement gravé sur le visage de Cherea.

Selon cette phrase: "Si nous mentons, c'est souvent sans le savoir. Je plaide non coupable", Cherea se défend immédiatement. C'est un bon pas pour récupérer la liberté qu'il veut. Il a trouvé une échappatoire à l'accusation de Caligula. Pour lui, les mensonges ne sont ni erreur, ni quelque chose qui doit être expliqué aux autres. C'est une forme de sa révolte métaphysique.

\section{CONCLUSION}

Les conclusions trouvées sont basées sur les résultats de l'analyse dans cette recherche. La chercheuse a trouvé une interprétation interprétative de l'absurdité du récit du drame. Camus a décrit comment l'absurdité se combine à la mort et à la révolte. Je peux donc en conclure que Camus veut expliquer où la mort est la limite extérieure d'une existence. Les êtres-humains ne peuvent pas simplement renoncer à l'absurdité et, pour maintenir cette existence, une révolte est nécessaire.

La chercheuse a remarqué ces perspectives comme le résultat d'une interprétation profonde selon laquelle, dans le drame de Caligula, Albert Camus était d'avis que le fait de juger l'absurdité était une chose absolue à laquelle l'homme devait faire face. Les principaux éléments et valeurs philosophiques de la pensée de Camus sont liés à la conscience, à la liberté et à la rébellion, qui feront plus tard ressortir plus profondément le sens de la liberté humaine.

\section{BIBLIOGRAPHIE}

Camus, Albert. 1951. L'Homme Révolté. Paris: Éditions Gallimard.

Djuharmie, E.K. dan Asep Juanda. 2004. Mari Bermain Drama. Jakarta: Regina

Endraswara, Suwardi. 2011. Metode Pembelajaran Drama. Jogyakarta : CAPS

Fitriana, Dina. 2016. Eksistensi Keberagaman: Studi Terhadap Pemikiran Ekistensialisme Soren Kierkegaard. Disertasi. Kridalaksana, Harimurti. 2008. Kamus Linguistik. Jakarta: Gramedia Pustaka Utama

Ratna, Nyoman Kutha. 2012. Teori, Metode, dan Teknik Penelitian Sastra.Yogyakarta: PustakaPelajar. Ricœur, Paul. 2012. Teori Interpretasi.Yogyakarta: IRCiSoD

Wellek, Rene dan Austin Warren. 2014. Teori Kesusastraan. Jakarta: Gramedia Pustaka Utama. 